\title{
Editorial
}

\section{Bioinspired materials: An emerging field of multidisciplinary research}

1 Cordt Zollfrank

Professor, Technische Universität München, Straubing, Germany

2 André Studart

Professor, ETH Zurich, Switzerland
3 Richard Weinkamer

Research Group Leader, Max Planck Institute of Colloids and Interfaces, Potsdam, Germany
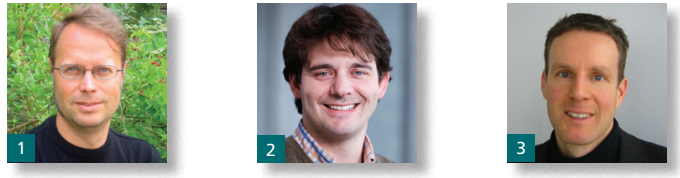

The development of novel advanced materials, where biological concepts and processing designs are adapted to solve engineering, civil and biomedical issues has attracted increasing interest in the scientific community. The idea of bioinspired materials design is to employ biological processing solutions, design strategies and hierarchical structures for the creation of advanced engineering and functional materials. Bioinspired materials engineering includes the following approaches: (a) using man-devised assembling techniques to replicate in a synthetic material the design principles of a biological structure, (b) replicating artificially the assembly process of natural materials (e.g. biomineralization) to obtain synthetic materials with unusual properties, and (c) using biological structures as templates for the creation of bioinspired materials with unusual structures and properties. It has to be noted that simply "copying" or "mimicking" of all involved biological processes and structures to build synthetic materials is not envisioned due to the wider range of chemical resources available in synthetic systems, the different boundary conditions and constraints of engineering applications and the extended processing times of natural assembly routes. Instead, biomimetic research should focus on replicating structural design principles from nature that might provide new, unusual solutions to engineering problems. The transfer of such design principles to synthetic materials requires the combined effort of natural sciences (physics, chemistry and biology) as well as engineering sciences (materials science, process engineering and bioengineering).

The hierarchical architecture, self-healing ability, adaptive behavior and intricate combination of organic matter, inorganic phases and possibly living cells observed in biological hybrid structures find no counterpart in today's artificial materials. Combining the structural design concepts of natural hybrid materials with the wealth of chemical compositions available in synthetic systems will possibly enable the assembly of bioinspired artificial materials exhibiting novel unusual properties. Hence, nature is an invaluable source of inspiration for entirely novel materials design and processing.

This motivated us to organize a symposium that would cover several fundamental aspects of this very interdisciplinary field. The symposium was held between the 12th and 15th September 2011 at the EUROMAT, in Montpellier, France, under the topic "New Concepts in Structural Materials", coordinated by Prof. Sybrand Van der Zwaag. After successful symposia on "Bioinspired Materials" in 2007 (Nuremberg, Germany) and 2009 (Glasgow, United Kingdom), we had now the opportunity to continue gathering scientists from all over the world to contribute to this highly emerging field. The symposium received more than 20 contributions from internationally renowned scientists. The submissions to the conference symposium were grouped into three sessions: Natural systems, Biomineralisation, and Bioinspired Films and Composites together with a joint poster session.

During and after the successful symposium a call for papers was launched to provide the participants with the opportunity to publish their presented work in this Themed Issue for Bioinspired, Biomimetic and Nanobiomaterials. We are pleased that we could attract a number of papers from the symposium participants. The submitted papers were grouped reflecting the original structure of the symposium. Thus, we are delighted that we are able to present three contributions dealing with natural systems, three papers from the field of bioinspired composites and two further manuscripts related to biomineralization.

Opening the contributions on natural systems, Fleck et al (2012) describe their latest findings and interpretation on the fracture 
behavior of Macadamia nutshells. By investigating the failure of shell specimens under tension and compression using computed microtomography and microscopy techniques, the authors show that the shell's strength is strongly affected by the outer and inner sutures, the vascular bundles, and the interfaces between cells or cell bundles in the shell. The interaction of propagating cracks with such microstructural features are thoroughly discussed, providing new insights into the factors leading to the unusually high strength and impact resistance of Macadamia shells.

In the second contribution, Haushahn et al (2012) direct their research to the fundamental understanding of junction between stems and branches in arborescent monocotyledons and columnar cacti, since both plant groups share distinctive anatomical features. The relevant type of tissues of the indented or necked attachment areas were investigated and comprehensively described with respect to their microstructural and mechanical features. The obtained results from the natural system are reported to have a great potential to improve joints in braided fiber-reinforced composites, which will result into the future development of a fully automated fabrication of biomimetic branched composites.

Keerl et al (2012) introduce the reader into the fascinating mechanical properties of spider silk produced by orb-weaving spiders. They analyzed the mechanical characteristics of dragline silk fibers of Araneus diadematus and compared the obtained values to artificially produced fibers of a recombinant silk protein. It was demonstrated that these "biotech" fibers exhibited mechanical properties comparable to other artificial silk fibers made from proteins with comparable molecular weight. The $\beta$-sheet content of the biotech fibers is lower and their orientation is poorer than in native dragline silks. The fabricated bioinspired silk fibers hold promise for engineering and biomedical applications.

This leads the reader to the second group of papers related to the fabrication and characteristics of bioinspired composites and coatings. In their manuscript Gruber and Zollfrank (2012) report the synthesis of noble metal nanoparticles on silica nanowire biotemplated from cellulose nanocrystals. The cellulose nanocrystals are excellent biotemplates to obtain nanotubular structures. Tuning the noble metal particle composition from pure $\mathrm{Au}$ and $\mathrm{Au} / \mathrm{Ag}$ nano alloys resulted in variation of the observed localized surface plasmon resonance. It was additionally shown that the biotemplated noble metal nanoparticle modified one-dimensional nanostructures exhibit a high potential as substrate structure for surface enhanced Raman spectroscopy.

Changing the subject from one-dimensional nanostructures to three-dimensional spherical systems (capsules), Grigoriev et al (2012) showed the latest development on the fabrication of microand nanocontainers for biomimetic self-healing and self-protecting functional materials coatings. This particular class of functional materials are able to regain their functionality after damage. The authors described and reviewed the art of container synthesis for biomimetic functional coatings by use of an emulsion based system. It was shown that successful incorporation of containers in the various types of functional coatings exhibited promising characteristics for the application in self-healing coatings.

The scientific collaboration between four different groups led to the investigation of the transformation of natural templates into purely inorganic materials. The paper of Van Opdenbosch et al (2012) reports on the conversion of the complex three-dimensional structure and replication of pomelo (Citrus maxima) peel into amorphous silica on several hierarchical levels. The results indicated that the gradient foam structure of the peel was entirely replicated from the millimeter to the nanometer scales. This work shows basic considerations on how to generate inorganic, temperature resistant gradient foam structure from complex low density polysaccharidebased natural templates.

The last two papers included in this Themed Issue are dealing with scientific questions from the field of biomineralization. In the first paper from this group, Seidel et al (2012) guide the reader through a detailed microstructural analysis using synchrotron three-dimensional small angle x-ray scattering for the analysis of bone. Owing to the complexity of the structure of bone a structural description on the various hierarchical levels has to be performed. The authors describe scanning small-angle x-ray scattering method for the reconstruction of the threedimensional habit of mineral platelets within the hard tissue bone. The presented reconstruction technique might be of general importance for the analysis of nanoparticle orientation in highly textured biomaterials.

This Themed Issue is concluded by the contribution of Griesshaber et al (2012) on another mineralized tissue, the tooth and jaw of the sea urchin Paracentrotus lividus. The teeth of the sea urchin have received attention from the bioinspired viewpoint due to a mechanism of continuous self-sharpening by chipping-off structural units. Using a combination of micro-tomography, scanning electron microscopy and electron backscatter diffraction to study in particular the crystal architecture, the authors show a systematic misorientation of the structural units by a few degrees. These misorientations occur on two different length scales and throughout the entire length of the tooth.

The Guest Editors wish to thank ICE publishing and $B B N$, that is, the Editor-in-Chief Kajal Mallick and Managing Editor Sohini Banerjee, for their continued support, giving us the opportunity to participate in the production process of this journal and to prepare this Themed Issue. We are also grateful to all contributors, reviewers and co-workers, who sometimes had to finish their work under tight time constraints. The Themed Issue as we can present it now reflects selected studies performed in the emerging field of Bioinspired Materials and certainly opens the discourse to be 
addressed in future conferences and publications. We hope that the selected contributions will add to the ongoing fruitful discussion on Bioinspired Materials already and that the reader will enjoy the presented Themed Issue as much as we did.

\section{REFERENCES}

Fleck C, Schüler P, Meinel D, Zaslansky P and Currey J (2012)

Vascular bundles dominate failure in Macadamia nutshells. Bioinspired, Biomimetic and Nanobiomaterials 1:67-76.

Griesshaber E, Goetz A, Howard L et al (2012) Crystal architecture of the tooth and jaw of P. lividus. Bioinspired, Biomimetic and Nanobiomaterials 1:133-140.

Grigoriev D, Haase M, Fandrich N, Latnikova A and Shchukin D (2012) Emulsion route in fabrication of micro- and nanocontainers for biomimetic self-healing and selfprotecting functional coatings. Bioinspired, Biomimetic and Nanobiomaterials 1:101-116.
Gruber S and Zollfrank C (2012) Noble metal nanoparticles on biotemplated nanowires. Bioinspired, Biomimetic and Nanobiomaterials 1:95-100.

Haushahn T, Schwager H, Neinhuis C, Speck T and Masselter T (2012) Plant ramifications inspire branched lightweight composites. Bioinspired, Biomimetic and Nanobiomaterials 1:77-81.

Keerl D and Scheibel T (2012) Characterization of natural and biomimetic spider silk fibers. Bioinspired, Biomimetic and Nanobiomaterials 1:83-94.

Seidel R, Gourrier A, Kerschnitzki M et al (2012) Synchrotron 3D small angle $\mathrm{x}$-ray scattering analysis of bone. Bioinspired, Biomimetic and Nanobiomaterials 1:123-132.

Van Opdenbosch D, Thielen M, Seidel R et al (2012) The pomelo peel and derived nanoreplica silica gradient foams. Bioinspired, Biomimetic and Nanobiomaterials 1:117-122. 
Downloaded by [] on [26/04/23]. Copyright $\odot$ ICE Publishing, all rights reserved. 